\title{
VARIACIONES EN LA RAZÓN C:N:P DEL MATERIAL SESTÓNICO RECOGIDO EN TRAMPAS DE SEDIMENTACIÓN EN EL EMBALSE DE CHARCO REDONDO (CÁDIZ)
}

\author{
Eduardo Sáez; Roberto García; Juan Lucena y F. Xavier Niell. \\ Departamento de Geología y Ecología. \\ Facultad de Ciencias. Universidad de Málaga. \\ Campus Universitario de Teatinos. 29071-MALAGA.
}

Keywords: C:N:P ratio, settling seston, allochthonous input

\begin{abstract}
VARIATIONS IN THE C:N:P RATIO IN THE MATERIAL COLLECTED B Y SEDIMENT TRAPS IN CHARCO REDONDO RESERVOIR (CÁDIZ).

The C:N:P ratio in the entrapped material by sediment traps positioned at different dephts during twelve exposure periods during two annual cycles shows clear deviations from the Redfield values (106:16:1).

There were no significant differences in the above ratio with depth. However clear differences appeared in the C:P and $\mathrm{N}: \mathrm{P}$ ratios between stratification and mixing periods. The phosphorus fractional composition shows that the residual phosphorus (=organic plus inert phosphorus) is the phosphorus form most abundant. From data of the C:N:P ratio and phosphorus fractional composition in the material collected, the dynamic of this recently constructed reservoir is discussed.
\end{abstract}

\section{INTRODUCCIÓN}

Las proporciones atómicas de los elementos $\mathrm{C}: \mathrm{N}: \mathrm{P}$ en la materia viva, y especialmente en los productores primarios, han sido motivo de numerosos trabajos, (UEHLINGER y BLOESCH, 1987; GÁLVEZ et al., 1990; TEZUKA, 1986; GÄCHTER y MARES, 1985), desde que REDFIELD et al. (1963) establecieron para fitoplancton marino vivo que dicha razón es de 106:16:1. El análisis estequiométrico del material sestónico recogido en trampas de sedimentación proporciona información acerca de la naturaleza y origen de la materia orgánica que sedimenta así como del elemento responsable de la limitación en el crecimiento fitoplanctónico.

En un embalse recientemente construido, como es el de Charco Redondo (Cádiz) (Figura 1), además de los factores que normalmente son los responsables de las variaciones de las proporciones C:N:P, los elevados valores de flujo neto de material particulado (GARCÍA et al., 1993) introducen una fuente de variabilidad adicional.

\section{MÉTODOS}

En la zona central del embalse, de unos 35 metros de profundidad, se dispusieron trampas de sedimentación, de forma cilíndrica, con un diámetro de $6.4 \mathrm{~cm}$ y $48 \mathrm{~cm}$ de altura, con lo que se consigue una relación $\mathrm{H} / \mathrm{D}=7.5$, suficiente para evitar la resuspensión del material atrapado (GÁLVEZ, 1990). Las trampas se situaron tal y como se esquematiza en la figura 2, con las profundidades referidas como alturas desde el fondo. El estudio se realizó desde Octubre de 1989 hasta Diciembre de 1992, con tiempos de exposición de las trampas que oscilaron entre uno y tres meses. El material recogido, tras eliminar parte del líquido sobrenadante, se homogeneizó mediante agitación, filtrándose una porción a través de un filtro de fibra de vidrio Whatman $\mathrm{GF} / \mathrm{F}$ (0.7 pm de diámetro medio de poro) con objeto de calcular los flujos de material. El resto se secó (24 horas a $105{ }^{\circ} \mathrm{C}$ ) para la realización de posteriores análisis. Las proporciones de carbono particulado total y nitrógeno particulado total se midieron en el material seco 


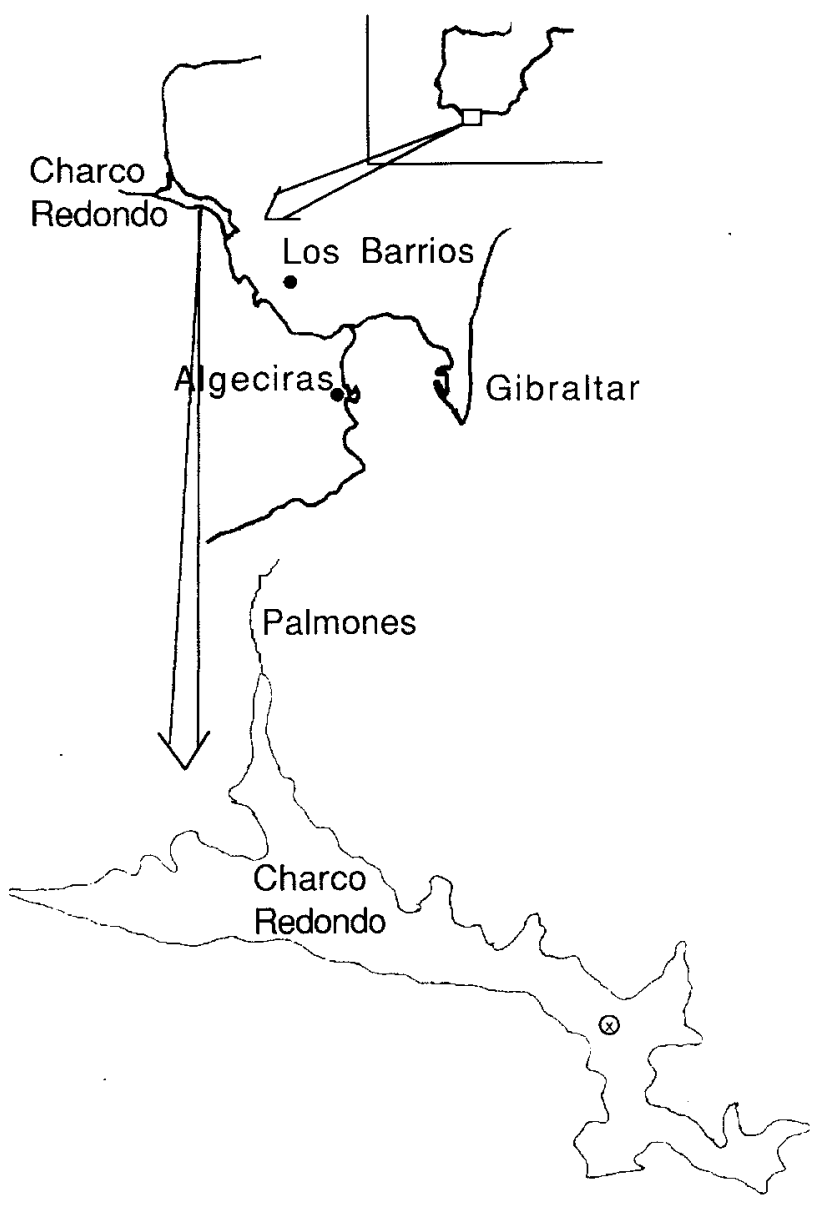

FIGURA 1.- Situación geográfica del embalse de Charco Redondo. Se señala con una cruz el lugar en que se dispusieron las trampas de sedimentación

FIGURE 1.- Geographic location of Charco Redondo reservoir. The station for sediment traps is showed with a cross.

mediante un Analizador Elemental Perkin-Helmer 240 C. El fósforo particulado total se midió, tras una digestión ácida con $\mathrm{HNO}_{3}-\mathrm{HClO}_{4}(5: 3)$ (SOMMERS \& NELSON, 1972), como ortofosfato (FERNÁNDEZ et al., 1985).

El fósforo lábilmente unido, el unido a hierro y a aluminio, y el unido a calcio se ha determinado en el periodo de mezcla y de estratificación mediante fraccionamiento según el método propuesto por HIELTJES \& LIJKLEMA (1980), seleccionándose muestras de fondo, profundidad intermedia y superficie. La fracción de fósforo residual, compuesta por fósforo orgánico más fósforo inerte, de difícil extracción, se estimó sustrayendo al fósforo total la suma de las tres fracciones inorgánicas mencionadas anteriormente.

\section{RESULTADOS Y DISCUSIÓN}

Con objeto de poner de manifiesto la existencia o no de variaciones, verticales y estacionales, entre los valores obtenidos de las relaciones $\mathrm{C}: \mathrm{N}, \mathrm{C}: \mathrm{P}$ y N:P (Tabla 1) se aplicó una prueba t-student. La misma prueba estadística se usó para comparar los valores medios de las razones de cada grupo de muestras con el valor esperado según Redfield.

No se encontraron diferencias significativas por profundidades para ninguna de las tres razones. Por el contrario, al comparar las muestras de mezcla y estratificación, las diferencias fueron significativas para el C:P y para el N:P a un $\alpha<0.001$, no encontrándose dicha diferencia para el $\mathrm{C}: \mathrm{N}$. Por otra parte, todos los valores resultaron significativamente distintos de los de Redfield $(\alpha<0.05)$.

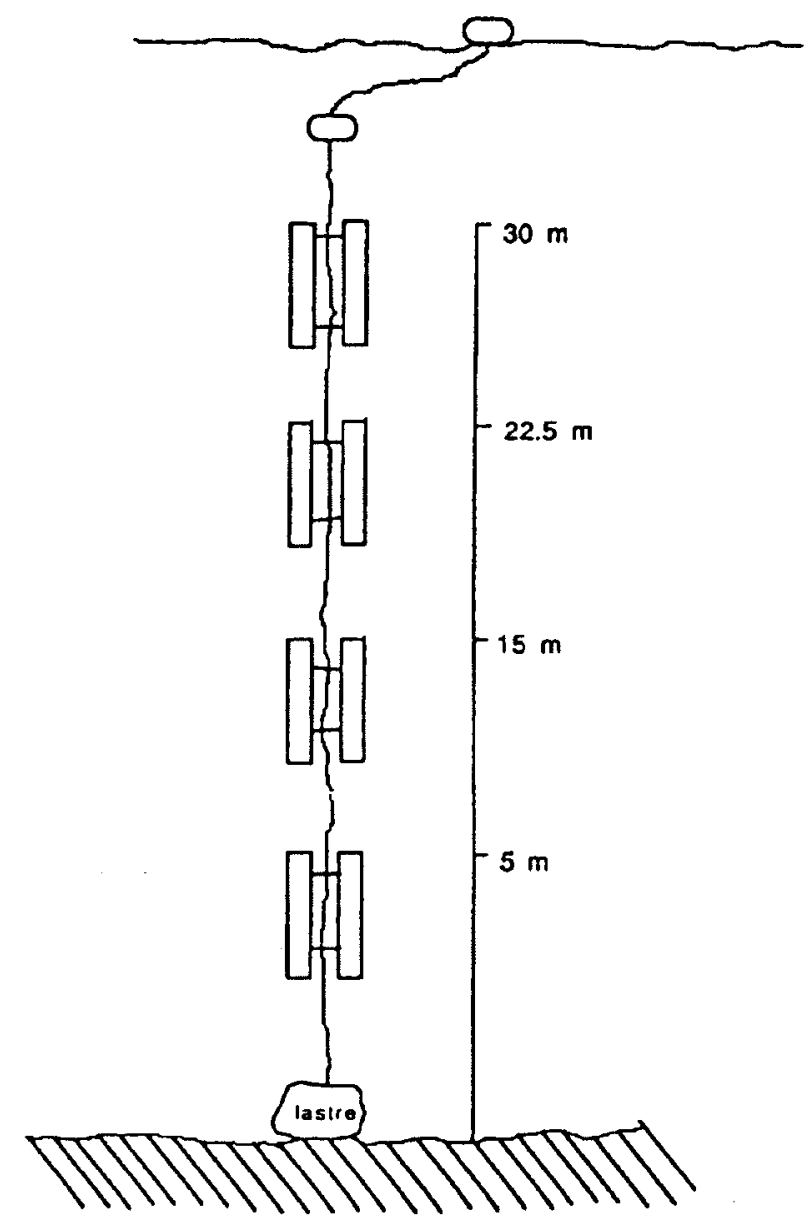

FIGURA 2.- Disposición de las trampas de sedimentación. FIGURE 2.- Sediment traps position in the water column. 
TABLA 1.- Valores medios de las razones C:N, C:P y N:P de cada grupo de muestras realizadas.

TABLE 1.- Average values of the C:N, C:P and N:P ratios from the different sample groups.

№ muest. $\quad$ Valor medio

$\begin{array}{ccc}\text { C:N (Redfield: 6,6) } & & \\ \text { Total } & 41 & 11,0 \pm 2,0 \\ \mathrm{~h}=30 \mathrm{~m} & 07 & 11,5 \pm 2,8 \\ \mathrm{~h}=22,5 \mathrm{~m} & 11 & 10,8 \pm 2,1 \\ \mathrm{~h}=15 \mathrm{~m} & 11 & 11,0 \pm 2,1 \\ \mathrm{~h}=5 \mathrm{~m} & 12 & 10,8 \pm 1,5 \\ \text { Estrat. } & 14 & 10,9 \pm 1,5 \\ \text { Mezcla } & 27 & 11,0 \pm 2,3\end{array}$

\begin{tabular}{ccc}
\hline C:P (Redfield: 106) & & \\
Total & 38 & $72,9 \pm 19,6$ \\
$\mathrm{~h}=30 \mathrm{~m}$ & 07 & $78,0 \pm 29,9$ \\
$\mathrm{~h}=22,5 \mathrm{~m}$ & 10 & $71,0 \pm 17,5$ \\
$\mathrm{~h}=15 \mathrm{~m}$ & 10 & $71,5 \pm 19,6$ \\
$\mathrm{~h}=5 \mathrm{~m}$ & 11 & $69,9 \pm 15,1$ \\
Estrat. & 14 & $92,8 \pm 14,2$ \\
Mezcla & 24 & $60,0 \pm 09,3$ \\
\hline
\end{tabular}

N:P (Redfield: 16)

$\begin{array}{lll}\text { Total } & 38 & 6,8 \pm 1,8 \\ \mathrm{~h}=30 \mathrm{~m} & 07 & 7,0 \pm 2,7 \\ \mathrm{~h}=22,5 \mathrm{~m} & 10 & 6,9 \pm 1,2 \\ \mathrm{~h}=15 \mathrm{~m} & 10 & 6,7 \pm 1,7 \\ \mathrm{~h}=5 \mathrm{~m} & 11 & 6,6 \pm 2,0 \\ \text { Estrat. } & 14 & 8,6 \pm 1,3 \\ \text { Mezcla } & 24 & 5,7 \pm 1,1\end{array}$

Las representaciones $\mathrm{C}$ vs $\mathrm{N}, \mathrm{C}$ vs $\mathrm{P}$ y N vs $\mathrm{P}$ (figuras 3, 4 y 5 ) muestran una desviación con relación a los valores dados por Redfield, resultado coincidente con lo encontrado por otros autores (JONES, 1976; GACHTER \& BLOESH, 1985; UEHLINGER \& BLOESH, 1987; GÁLVEZ, 1990).

La razón $\mathrm{C}: \mathrm{N}$ no presenta variaciones significativas ni con la profundidad ni con la estacionalidad, permaneciendo constante y cercana a 11 , valor significativamente mayor del de Redfield (Figura 3). Esta desviación es atribuible a la mineralización del material autóctono in situ ya que es característico de la materia orgánica alóctona tener un índice C:N mucho mayor, de hasta 50 en sistemas 1acustres en general (WETZEL, 1980).

En Charco Redondo los valores de clorofila $\boldsymbol{a}$ total son

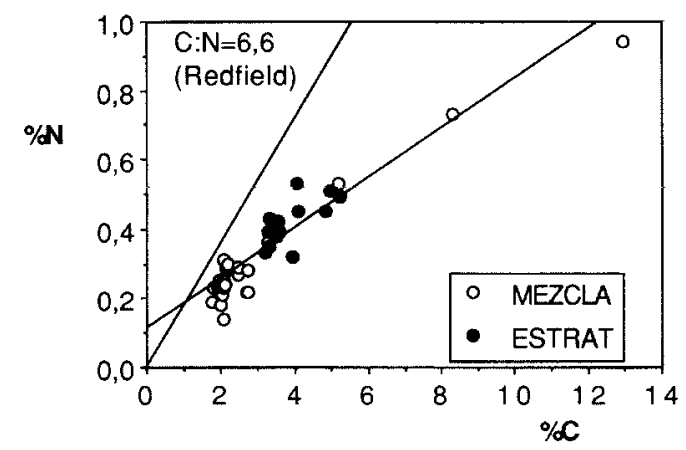

Fig. 3

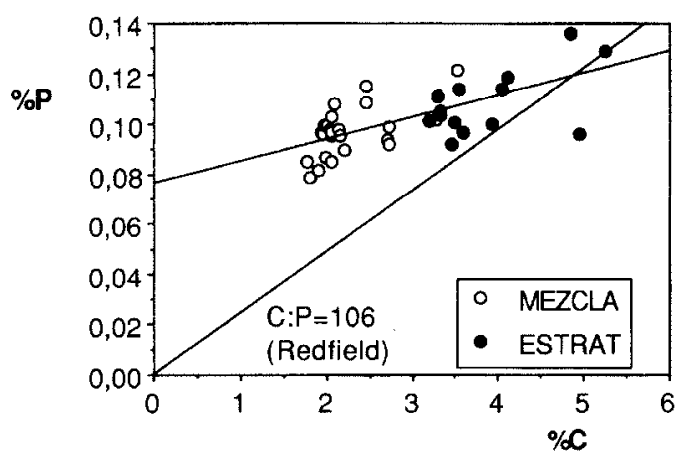

Fig. 4

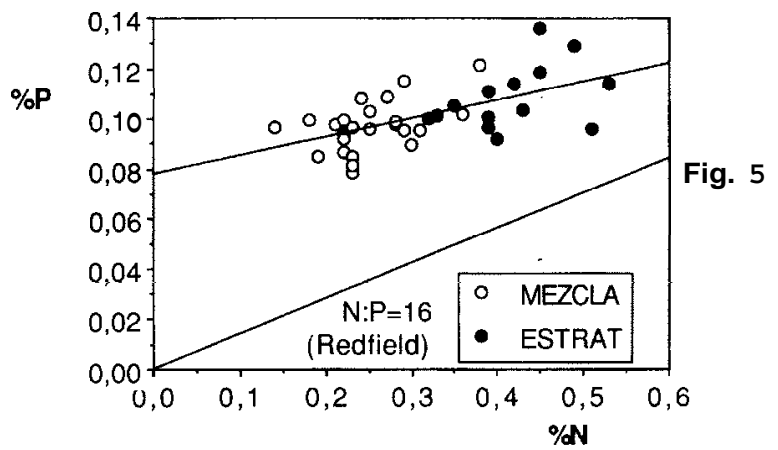

FIGURAS 3, 4, y 5.- Representación de las proporciones de C vs N, C vs $\mathrm{P}$ y $\mathrm{N}$ vs $\mathrm{P}$ en la etapa de mezcla y de estratificación, indicando la recta correspondiente a la razón de Redfield así como el ajuste lineal para cada representación.

FIGURES 3, 4 y 5.- Percentages of $\mathrm{C}$ vs $\mathrm{N}, \mathrm{C}$ vs $\mathrm{P}$ and $\mathrm{N}$ vs $\mathrm{P}$ in mixing and stratification periods. Lines represents the ratio calculated from data and the redfield ratio.

bajos durante todo el año, oscilando entre $2 \mu \mathrm{g} / \mathrm{l}$ (mezcla) y $7 \mu \mathrm{g} / 1$ (estratificación), y los aportes de material alóctono son muy importantes y variables en función de la torrencialidad de las lluvias (GARCÍA et al., 1993). El hecho de que las proporciones de $\mathrm{C}$ y de $\mathrm{N}$ del material recogido en 
las trampas sean en estratificación (cuando la producción primaria es mayor y los aportes alóctonos muy pequeños) más altas que en mezcla (cuando la producción es mínima y los aportes alóctonos máximos), confirma el origen fundamentalmente autóctono de la materia orgánica retenida en las trampas.

De hecho, existe un grupo de puntos en la representación $\mathrm{C}$ vs $\mathrm{N}$ (figura 3). que aun siendo de la etapa de mezcla, presentan los máximos valores de $\mathrm{C}$ y de $\mathrm{N}$, y muestran el máximo alejamiento de la recta $\mathrm{C}: \mathrm{N}=6,6$. Dichos puntos corresponden a un periodo de lluvias intensas en que el aporte de materiales alóctonos fué elevado (GARCÍA et al., 1993) y la materia orgánica de igual origen debió tener un peso importante.

Según GACHTER \& BLOESH (1985), GACHTER \& MARES (1985), TEZUKA (1986) y GÁLVEZ (1990), la razón $\mathrm{C}: \mathrm{P}$ disminuye con la profundidad por motivos aún no bien establecidos. En Charco Redondo no se da esta variación de forma significativa, aunque se observa una ligera tendencia en este sentido. Sin embargo se encontraron variaciones estacionales, siendo más altas las razones $\mathrm{C}: \mathrm{P}$ y N:P en estratificación que en mezcla. Esto es, la proporción de fósforo disminuye menos que la de nitrógeno y la de carbono, con lo que la nube de puntos no tiende a acercarse al origen de coordenadas, sino que existe un valor umbral de fósforo para proporciones de carbono y de nitrógeno iguales a cero del $0,08 \%$ aproximadamente (figuras 4 y 5), valor que corresponde al punto de corte en ordenadas de la función obtenida mediante regresión lineal tanto en $\mathrm{C}$ vs $\mathrm{P}$ como en $\mathrm{N}$ vs P. Este valor teórico puede atribuirse a la proporción de fósforo del seston en ausencia de materia orgánica. La elevada tasa de sedimentación del material sestónico, con una alta proporción de materia inorgánica, que es debida a la inestabilidad de los materiales del terreno recientemente inundado (GARCÍA et al., 1993) puede conferir al fósforo inorgánico un peso suficiente como para enmascarar variaciones en las razones atómicas en que está involucrado el mismo e impedir la detección de patrones de variación específicos con la profundidad.

El estudio fraccionado del fósforo total (tabla 2, figura 6), revela una proporción de las fracciones de fósforo lábilmente unido y del apatítico menor del $1 \%$, alrededor de un $20 \%$ del unido a hierro y a aluminio, y de un $80 \%$ del residual. Esta elevada proporción de fósforo residual sería atribuible, en principio, mayoritariamente a fósforo orgánico (PETTERSSON, BOSTRÖM \& JACOBSEN, 1988; FURUMARI \& OHGAKI, 1982) pero en nuestro caso, esto supondría una proporción de fósforo orgánico muy elevada, restando una proporción de fósforo inorgánico menor de la esperada según la estimación anterior del $0.08 \%$. Además, no existe correlación entre ambas variables (fósforo residual y materia orgánica) por lo que una parte importante de esta elevada proporción de fósforo residual debe ser fósforo inerte que ha quedado sin extraer tras los distintos tratamientos. Por otra parte, el suelo de las zonas

TABLA 2.- Proporciones de las distintas fracciones de fósforo particulado, cantidad total del mismo y proporción de materia orgánica en el seston a tres profundidades distintas en un periodo de mezcla y en otro de estratificación.

TABLE 2.- Fractional composition of phosphorus (in percentages), total phosphorus and percentages of organic matter in the material collected in mixing and stratification periods at three different depths.

\begin{tabular}{|c|c|c|c|c|c|c|c|}
\hline FECHA & $h(\mathrm{~m})$ & $\begin{array}{c}\% \mathrm{P} \\
\text { Lábil }\end{array}$ & $\begin{array}{c}\% \mathrm{P} \\
\mathrm{Fe}, \mathrm{Al}\end{array}$ & $\begin{array}{l}\% \mathrm{P} \\
\mathrm{Ca}\end{array}$ & $\begin{array}{l}\% \mathrm{P} \\
\text { res }\end{array}$ & $\begin{array}{l}\text { Total(100\%) } \\
\text { (mgP/gP.S.) }\end{array}$ & $\% \mathrm{MO}$ \\
\hline \multirow[t]{6}{*}{ Marzo-91 } & 22.5 & 0.30 & 19.75 & 0.51 & 79.44 & 0.91 & 15.1 \\
\hline & & 0.30 & 18.80 & 0.51 & 80.39 & & \\
\hline & 15 & 0.27 & 18.37 & 0.51 & 80.85 & 0.97 & 19.9 \\
\hline & & 0.18 & 15.34 & 0.50 & 83.98 & & \\
\hline & 5 & 0.31 & 18.88 & 0.47 & 80.34 & 0.92 & 9.6 \\
\hline & & 0.28 & 18.20 & 0.53 & 80.99 & & \\
\hline \multirow[t]{6}{*}{ Julio-91 } & 30 & $0 . \overline{40}$ & 25.85 & 0.68 & 73.07 & 1.23 & 13.5 \\
\hline & & 0.47 & 22.89 & 0.64 & 76.00 & & \\
\hline & 15 & 0.22 & 17.68 & 0.61 & 81.49 & 0.94 & 10.9 \\
\hline & & 0.20 & 16.23 & 0.57 & 83.00 & & \\
\hline & 5 & 0.16 & 12.40 & 0.68 & 86.76 & 0.92 & 13.2 \\
\hline & & 0.40 & 15.93 & 0.69 & 82.98 & & \\
\hline
\end{tabular}



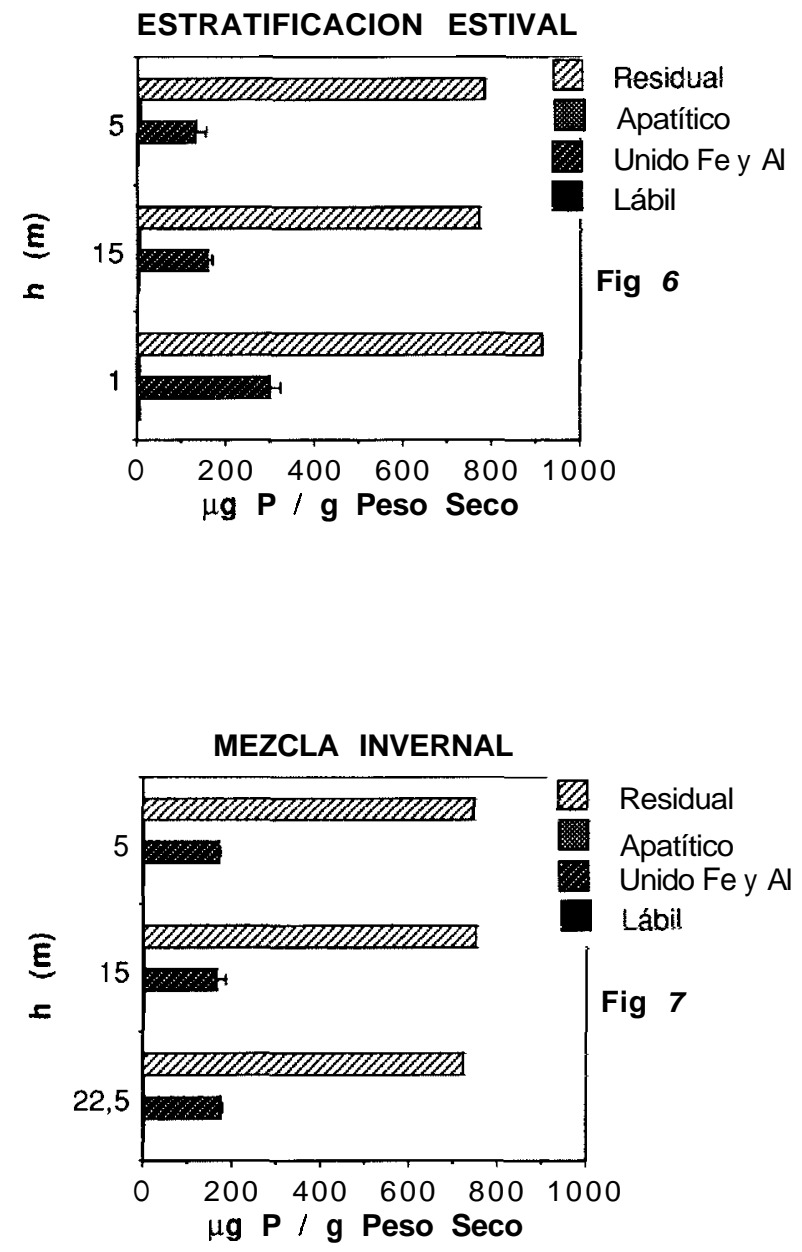

FIGURAS 6 y 7.- Proporciones de fósforo tras el fraccionamiento en muestras correspondientes a las etapas de mezcla y de estratificación. FIGURES 6 y 7.- Fractional composition of phosphorus in the material collected in the traps in stratification and mixing periods.

de los alrededores presenta una composición en fósforo total de entre 0.34 y $0.65 \mathrm{mgP} / \mathrm{g}$ P.S. (CARREIRA, comunicación personal), sensiblemente menor que la del seston (de alrededor de $1 \mathrm{mgP} / \mathrm{gP}$.S.), lo que sugiere que la diferencia (de aproximadamente $0.5 \mathrm{mgP} / \mathrm{g}$ P.S.) se debe a fósforo orgánico, que supondría más de la mitad del fósforo residual.

Aunque las proporciones de $\mathrm{C}, \mathrm{N}$ y $\mathrm{P}$ del material sestónico de las trampas no son elevadas (figuras 3, 4 y 5), sí lo son los valores absolutos de materia orgánica que sedimenta ya que el flujo sestónico es excepcionalmente alto, no bajando de 10 gramos de seston por metro cuadrado y día lo que supone un flujo de más de $1 \mathrm{~g} \mathrm{~m}^{-2}$ día-' de materia orgánica y más de $10 \mathrm{mg}$ de $\mathrm{PT} \mathrm{m}^{-2}$ día $^{-1}$ (GARCÍA et al., 1993). Este flujo de fósforo hacia el hipolimnion es un orden de magnitud superior al estimado en sistemas oligotróficos como el lago Mirror (USA) (CARACO, COLE \& LIKENS, 1992) e incluso a sistemas eutróficos como el embalse de Río Verde (Málaga), donde el flujo de fósforo en el hipolimnion oscila alrededor de $1 \mathrm{mg} \mathrm{m}{ }^{2}$ día-' (GÁLVEZ, 1990). Esto, unido al origen fundamentalmente autóctono de dicha materia orgánica, puesto anteriormente de manifiesto, y considerando los bajos valores de clorofila que se alcanzan en el agua del embalse, nos lleva a postular que la producción primaria debe ser elevada y de ciclo rápido. De forma que se produce una rápida incorporación por parte del fitoplancton del ortofosfato liberado, que se encuentra a concentraciones no detectables (LUCENA, comunicación personal). Dicha liberación puede ser consecuencia de la mineralización de la materia orgánica autóctona (regeneración) y/o solubilización del fósforo unido a hierro y a aluminio (que representa una proporción considerable del fósforo total) durante la anoxia del final de la época de estratificación (renovación) y que podría representar una entrada importante de fósforo al sistema que permitiría soportar una mayor biomasa. Este proceso de eutrofización, que puede verse limitado por la poca penetración de la luz motivada por los altos valores de material particulado en suspensión que presenta el embalse (GARCÍA et al., 1993), es previsible que avance a medida que la cuenca vaya consolidándose.

\section{CONCLUSIONES}

El origen de la materia orgánica sestónica es fundamentalmente autóctono en contra de lo que parecen indicar los bajos valores de biomasa fitoplanctónica y los altos flujos de material alóctono de escorrentía, debiendo existir una producción primaria importante.

La proporción de fósforo inorgánico del material sedimentado (estimado en un $0.08 \%$ ) debe enmascarar variaciones en las razones $\mathrm{C}: \mathrm{P}$ y $\mathrm{N}: \mathrm{P}$ con implicaciones en el funcionamiento global del embalse, en el que los factores no biológicos juegan un papel fundamental.

\section{AGRADECIMIENTOS}

Este trabajo ha sido realizado con la ayuda económica de la Dirección General de Obras Hidráulicas y el Ministerio de Educación y Ciencia mediante el proyecto NAT900335 


\section{BIBLIOGRAFÍA}

CARACO, N.F., J.J.COLE, \& G.E. LIKENS, 1992. New and recycled primary production in an oligotrophic lake: insighyts for summer phosphorus dynamics. Limnol. Oceanogr., 37 (3): 590-602.

FERNÁNDEZ, J. A., F. X. NIELL \& J. LUCENA, 1985. A rapid and sensitive automated determination of phosphate in natural waters. Limnol. Oceanogr. 30: 227230.

FURUMARI, H. \& S. OHGAKI, 1982. Fractional composition of phosphorus forms in sediments related to release. Wat. Sci. Tech., 14: 215-226.

GÁLVEZ, J. A., 1990. Sedimentación hiogénica en el embalse de La Concepción (Málaga). Aproximación a un modelo para sistemas acuáticos continentales. Tesis Doctoral, Universidad de Málaga. 214pp.

GARCÍA R., E. SÁEZ, J. LUCENA, F. X. NIELL \& J. A. GÁLVEZ, 1993. Sedimentation patterns in a recient constructed reservoir (Charco Redondo, Los Barrios, Cádiz, Spain), 1992. Verh. Internat. Verein. Limnol., 25: 1223-1226.

GÄTCHER, R. \& J. BLOESCH, 1985. Seasonal and vertical variation in the $C: P$ ratio of suspended and settling seston of lakes. Hydrobiologia, 128:193-200.

GÄTCHER, R. \& A. MARES, 1985. Does settling seston release soluble reactive phosphorus in the hypolimnion of lakes?. Limnol Oceanogr., 30:364-371.

HIELTJES, A. H. M. \& L. LIJKLEMA, 1980. Fractionation of inorganic phosphate in calcareous sediments. $\mathbf{J}$. Environ. Qual., 9: 405-407.
JONES J. G., 1976. The microbiology and decomposition of seston in open water and experimental enclosures in a productive lake. J. Ecol., 64: 241-278.

PETTERSSON, K., BOSTRÖM, B. \& JACOBSEN, O.S., 1988. Phosphorus in sediments-speciation and analysis. Hydrohiologia, 170: 91-101.

REDFIELD, A. C., B. H. KETCHUM \& F. A. RICHARDS, 1963. The influence of organisms on the composition of sea-water. In: HILL, M. N. (Ed): The Sea. 2: 26-77. Interscience.

SOMMERS, L. E. \& D. W. NELSON, 1972. Determination of total phosphorus by a rapid perchloric acid procedure. Soil Sci. Soc. Amer. Proc., 36: 902-904.

TEZUKA Y., 1986. Does the seston of lake Biwa release dissolved inorganic nitrogen and phosphorus during aerobic decomposition?. Its implication for eutrophication. Ecol. Res., 1: 293-302.

UEHLINGER, U. J. \& BLOESCH, 1987. Variation in the $\mathrm{C}: \mathrm{P}$ ratio of suspended and settling seston and its significance for P uptake calculations. Freshwater biology, 17: 99-108.

UEHLINGER, U., P. BOSSARD, J. BLOESCH, H. R. BURGI, \& H. BÜHRER, 1984. Ecological experiments in limnocorrals: methodological problems and quantification of the epilimnetic phosphorus and carbon cycles. Verh. Internat. Verein. Limnol., 22:163-171.

WETZEL, R.G., 1980. Limnología. Ed. Omega, Barcelona. $679 \mathrm{pp}$. 